\title{
Pubic Majocchi's Granuloma Unresponsive to Itraconazole Successfully Treated with Oral Terbinafine
}

\author{
Efstathios Rallis $^{a} \quad$ Alexandros Katoulis $^{b} \quad$ Dimitrios Rigopoulos $^{b}$

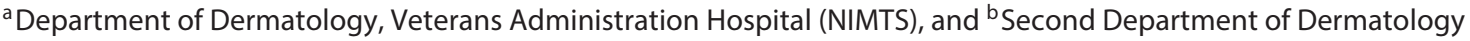 \\ and Venereology, 'Attikon' General University Hospital, Athens, Greece
}

\section{Key Words}

Majocchi's granuloma · Fungal folliculitis - Granuloma

trichophyticum · Itraconazole · Terbinafine · Pubic area

\begin{abstract}
Majocchi's granuloma (MG) is an uncommon deep fungal infection. It is usually caused by Trichophyton rubrum and may develop in any hair-bearing skin, commonly on the face and the extremities. We present a 27-year-old female with MG of the pubic area treated unsuccessfully with itraconazole capsule $100 \mathrm{mg}$ for 4 weeks. The medication was discontinued and switched to terbinafine, which proved efficacious. The pubic tinea clinically presented in conjunction with fever, lymphadenopathy, inflammatory plaques, follicular lesions and subcutaneous nodules. It was considered that the fungal infection was initially transmitted from skin to skin during sexual practices with her husband. The repeated shaving of her pubic area, the misdiagnosis as bacterial infection and the use of topical corticosteroid on a preexistent tinea probably predisposed the patient to MG.
\end{abstract}

(c) 2015 S. Karger AG, Basel
(C) 2015 S. Karger AG, Basel

2296-9195/15/0013-0111\$39.50/0

\section{Introduction}

Majocchi's granuloma (MG), also referred to as fungal folliculitis, granuloma trichophyticum or nodular granulomatous perifolliculitis, is an uncommon deep fungal infection. It is most commonly caused by Trichophyton rubrum. MG may appear in any hair-bearing skin area, most often on the scalp, the face or the extremities [1]. We present a case of MG on the pubis of a young female.

\section{Case Report}

A 27-year-old, immunocompetent woman was referred to us for a painful, inflammatory plaque of the pubic area of 3 months' duration (fig. 1). On clinical examination, a sharply demarcated, dusky red, swollen plaque with subcutaneous nodules and cysts, covered by yellowish crusts, was observed. Two smaller, annular, scaly plaques could also be seen on the left thigh. In addition, inguinal lymphadenopathy and a fever of $38.3^{\circ} \mathrm{C}$ were present. History revealed that the patient used to shave this area during the past years. Previous treatments with amoxicillin, ciprofloxacin and topical betamethasone valerate with fusidic acid had been unsuccessful.

Direct examination and culture of bacterial swabs were negative. In contrast, direct examination of a $20 \% \mathrm{KOH}$ preparation

\section{KARGER 125}

E-Mail karger@karger.com www.karger.com/sad
Dr. Efstathios Rallis

11 Pafsaniou str

GR-11635 Athens (Greece)

E-Mail efrall@otenet.gr 


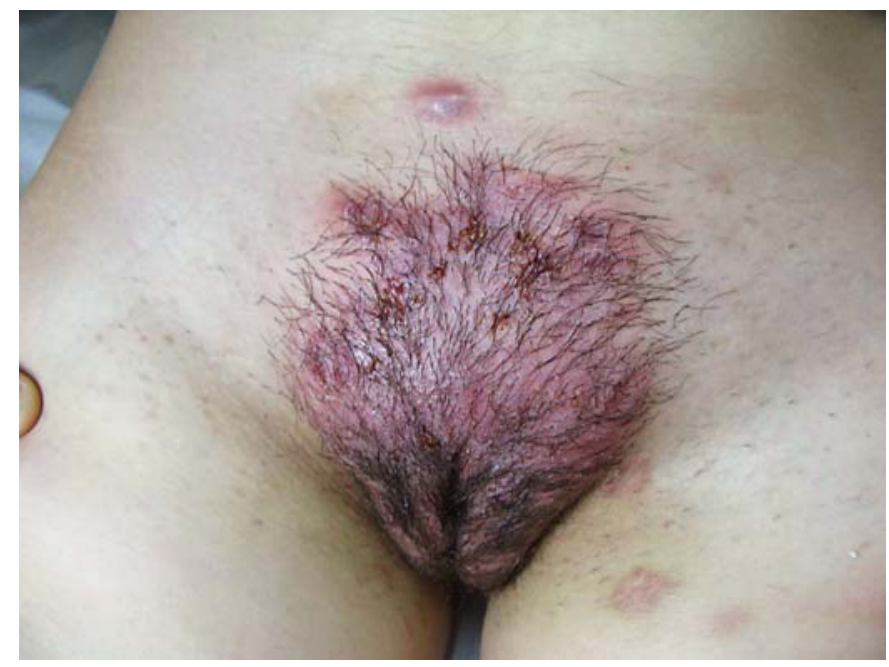

Fig. 1. Impetiginization of inflammatory plaques and subcutaneous nodules are seen in the pubic area. Two small, annular, scaly plaques can also be seen on the left thigh.

was positive. Cultures of scales and pus taken from the lesions revealed Trichophyton rubrum. Routine blood tests were within the normal range. HIV testing was negative. She did not consent to skin biopsy because of severe pain. Clinical examination of her husband showed the presence of tinea cruris of 1 year's duration. Mycological examination of the scales also revealed the presence of T. rubrum.

Both patients were treated with itraconazole $100 \mathrm{mg}$ once daily for 4 weeks, and miconazole nitrate cream twice daily. On the follow-up visit, the husband responded very well, but the wife's improvement was not satisfactory. Painful inflammatory nodules of the pubic area were still present. She also reported dry mouth after itraconazole initiation. Terbinafine was started at a dose of $250 \mathrm{mg}$ once daily for 2 months. At the end of the treatment, her general condition was excellent, inflammatory nodules and cysts had subsided, and only postinflammatory hyperpigmentation was evident (fig. 2). Mycological examination was negative. She reported no adverse events during the treatment course with terbinafine.

\section{Discussion}

MG was first described by Prof. D. Majocchi as 'granuloma tricofitico' [2]. Unlike inflammatory tinea or kerion, which result from a hypersensitivity reaction to an infection induced by dermatophytes, MG usually starts as a suppurative folliculitis that may terminate in a granulomatous reaction [3].

There are two clinical types of MG. The follicular type is frequently seen in young women who shave their legs [4], as in our patient, or following local immunosuppres-

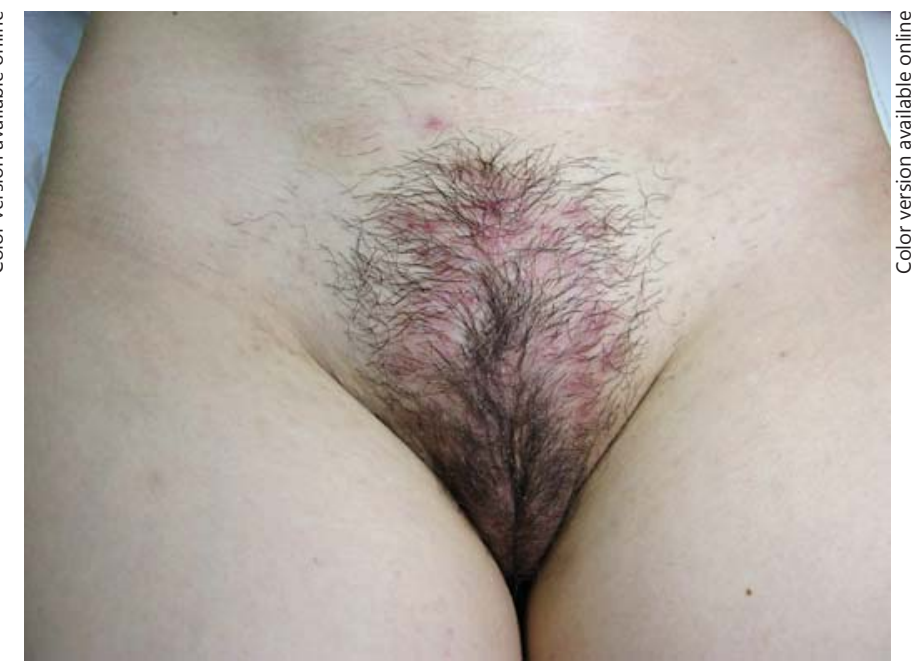

Fig. 2. The lesions subsided after continuous administration of terbinafine for 2 months.

sion after prolonged use of topical corticosteroids or calcineurin inhibitors [5]. The subcutaneous type occurs in immunocompromised hosts [1] and is manifested by plaques, subcutaneous nodules and abscesses. Mycologic examination is necessary to identify the responsible organism. Histological examination has proven helpful to establish the diagnosis.

Treatment of MG includes systemic antifungals, such as terbinafine and itraconazole. Topical antifungal treatment administered as monotherapy is ineffective because it is not able to penetrate through the dermis and/or the subcutaneous tissue.

The clinical presentation of our case included inflammatory plaques, follicular lesions and subcutaneous nodules. Localization of MG in the pubic area is highly unusual. To our knowledge, this is probably the first case of pubic MG to be reported in the literature. Skin-to-skin transmission during sexual practices should be considered in our patient. Repeated shaving of the pubic area, misdiagnosis as a bacterial infection and the use of a topical corticosteroid on a preexisting tinea may also have played a causal role.

In our patient, itraconazole $100 \mathrm{mg}$ once daily administered for 4 weeks proved ineffective. Steiner et al. [6] reported a heart transplant patient under prednisone, azathioprine and tacrolimus who developed a widespread T. rubrum infection presenting as MG. This fungal infection proved unresponsive to an 8-week regimen with oral itraconazole $100 \mathrm{mg}$ b.i.d. 
Oral terbinafine for 4-6 weeks is considered the treatment of choice for MG. However, in a recently published case, an immunocompetent man with an MG refractory to itraconazole and oral terbinafine was treated successfully with voriconazole [7].

The best results are probably achieved by a combination of systemic and topical antifungal therapy [1]. This therapeutic approach proved to be beneficial also in the present case.

\section{Statement of Ethics}

Both patients have provided their informed consent for the publication of their cases, and the study complies with human rights.

\section{Disclosure Statement}

The authors have no conflict of interest to declare.

\section{References}

1 Schwartz R: Majocchi granuloma (updated Oct 10, 2014). http://emedicine.medscape. com/article/1092601 (accessed Jan 7, 2015).

2 Majocchi D: Sopra una nuova trichofizia (granuloma tricofitico): studi clinici e micologici. Bull R Acad Med Roma 1883;9:220223 .

3 Arenas R, Toussaint S, Isa-Isa R: Kerion and dermatophytic granuloma. Mycological and histopathological findings in 19 children with inflammatory tinea capitis of the scalp. Int J Dermatol 2006;45:215-219.

4 Coelho WS, Diniz LM, Sousa Filho JB, Castro CM: Case for diagnosis. Granuloma trichophyticum (Majocchi's granuloma) (in Portuguese). An Bras Dermatol 2009;84:85-86.

5 Rallis E, Koumantaki-Mathioudaki E: Pimecrolimus induced tinea incognito masquerading as intertriginous psoriasis. Mycoses 2008;51:71-73.
6 Steiner UC, Trüeb RM, Schad K, et al: Trichophyton rubrum-induced Majocchi's granuloma in a heart transplant recipient. A therapeutic challenge. J Dermatol Case Rep 2012;6: $70-72$.

7 Liu HB, Liu F, Kong OT, et al: Successful treatment of refractory Majocchi's granuloma with voriconazole and review of published literature. Mycopathologia 2015, Epub ahead of print. 